\section{Die Stellung des ärztlichen Leiters im Medizinischen Versorgungszentrum}

\section{Einführung}

Die ärztliche Kooperationsform des Medizinischen Versorgungszentrums (MVZ) erfreut sich seit ihrer Einführung durch das GKV-Modernisierungsgesetz vom 14.11.2003 ${ }^{1}$ in der Ärzteschaft bis heute steigender Beliebtheit. ${ }^{2}$ Als Begründung für die Wahl des MVZ als Kooperationsform werden regelmäßig die Sicherung der Zukunftsfähigkeit der Praxis und die Verringerung des Investitionsrisikos für den einzelnen Arzt - insbesondere in den kapitalintensiven Methodenfächern - wie auch die erweiterte Möglichkeit zur Anstellung von Ärzten sowie die Nutzung von Synergieeffekten, insbesondere der Entlastung des einzelnen Arztes von Verwaltungsaufgaben, genannt. ${ }^{3}$ Dabei geht die Beliebtheit des MVZ auch mit einer zunehmenden Bereitschaft der Ärzteschaft zur Tätigkeit in der Anstellung einher. Während zur Einführung der Kooperationsform nur 144 Vertragsärzte und 107 angestellte Ärzte in MVZ tätig waren, praktizierten zum Jahresende 2019 bereits 1675 Vertragsärzte und 20212 angestellte Ärzte in 3539 MVZ mit weiter steigender Tendenz und ohne Berücksichtigung der zahlreichen zahnärztlichen MVZ. ${ }^{4}$ Schon aufgrund dieser erheblichen Bündelung ärztlicher Tätigkeit nehmen Medizinische Versorgungszentren heute eine tragende Rolle im Rahmen der Patientenversorgung ein und stellen eine beliebte Kooperationsform in der Ärzteund Zahnärzteschaft dar.

1 Gesetz zur Modernisierung der Gesetzlichen Krankenversicherung vom 14.11.2003, BGBI. 2003 I S. $2190 \mathrm{ff}$.

2 Kassenärztliche Bundesvereinigung (KBV), Entwicklung der Medizinischen Versorgungszentren, S. 3, 10, aufrufbar unter https:// www.kbv.de/media/sp/mvz_entwicklungen. pdf, letzter Abruf am 01.09.2021.

3 KBV, 4. MVZ Survey der KBV, S. 25 f., aufrufbar unter https://www.bmvz.de/wp-content/ uploads/2012/12/4_MVZ_Survey.pdf, letzter Abruf am 01.09.2021.

$4 \quad \mathrm{KBV}$, Entwicklung der Medizinischen Versorgungszentren, S. 3, 5, aufrufbar unter https:// www.kbv.de/media/sp/mvz_entwicklungen. pdf, letzter Abruf am 01.09.2021.
Jedem MVZ steht - nach Vorgabe des $§ 95$ Abs. 1 S. 2 SGB V - zwingend ein ärztlicher Leiter vor. Vor dem Hintergrund einer konstanten Zunahme der MVZ-Gründungen lohnt sich eine eingehendere Betrachtung von dessen Stellung und Verantwortungsbereich im MVZ und deren rechtlichen Grundlagen sowie möglichen persönlichen Haftungsrisiken - auch im Lichte der aktuellen Rechtsprechung. Die disziplinarischen Risiken des ärztlichen Leiters gegenüber der Kassenärztlichen Vereinigung (KV) zeigt dabei exemplarisch ein aktueller Gerichtsbescheid des Sozialgerichts (SG) München aus diesem Jahr.

\section{Funktion und Status des ärztlichen Leiters}

Für den Betrieb des MVZ ist die Bestellung eines ärztlichen Leiters zwingend erforderlich, der ärztliche Leiter ist bereits im Rahmen der Beantragung der Zulassung als MVZ gegenüber der KV zu benennen. ${ }^{5}$ Die Verpflichtung zur Bestellung eines ärztlichen Leiters ergibt sich dabei zunächst aus dem eindeutigen Wortlaut des $§ 95$ Abs. 1 S. 2 SGB V:

„Medizinische Versorgungszentren sind ärztlich geleitete Einrichtungen, in denen Ärzte, die in das Arztregister nach Absatz 2 Satz 3 eingetragen sind, als Angestellte oder Vertragsärzte tätig sind. " [sämtliche Hervorhebungen durch den Verfasser]

Der Wortlaut des $§ 95$ Abs. 1 S. 2 SGB V beschreibt das MVZ als ärztlich geleitete Einrichtung, der ärztliche Leiter muss infolgedessen zwingend selbst Arzt sein. Weiterhin erfordert das Gesetz die Eintragung der in dem MVZ tätigen Ärzte in das Arztregister der zuständigen KV - dies trifft auch auf den ärztlichen Leiter zu. Als Eintragungserfordernis hierzu muss der Betreffende zumindest als Arzt approbiert sein

5 Exemplarisch KV Westfalen-Lippe, Antrag auf Zulassung eines Medizinischen Versorgungszentrums, S. 13, aufrufbar unter https://www. kvwl.de/arzt/sicherstellung/antrag/pdf_zulas sung/arz_psy/mvz_antrag.pdf, letzter Abruf am 01.09.2021.

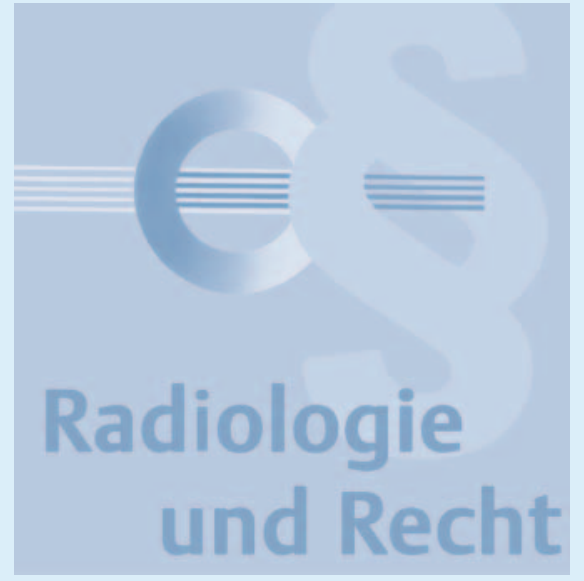

und über eine abgeschlossene Facharztausbildung verfügen, §95a Abs. 1 SGB V.

Nach § 95 Abs. 1 S. 3 SGB V muss der ärztliche Leiter weiterhin selbst in dem MVZ als angestellter Arzt oder Vertragsarzt tätig sein - der Tätigkeitsumfang muss sich dabei auf mindestens eine halbe Arztstelle belaufen. ${ }^{6}$ Dieses Erfordernis erwächst aus der besonderen Verantwortung des ärztlichen Leiters für die medizinischen Abläufe, deren Wahrnehmung die tatsächliche Einwirkungsmöglichkeit auf die medizinisch-ärztlichen Prozesse innerhalb des MVZ in Form ärztlicher Präsenz zwingend erfordert. ${ }^{7}$ Das Bundessozialgericht (BSG) stellt hierzu ausdrücklich fest:

"[...] Hinreichende tatsächliche Einwirkungsmöglichkeiten hat ein Arzt nur dann, wenn er selbst in die Arbeitsabläufe eingebunden ist und aus eigener Anschauung das Verhalten der Mitarbeiter beurteilen kann. Dass der ärztliche Leiter in der Geschäftsführung des MVZ

6 BSG, Urteil vom 19. Oktober 2011, Az. B 6 KA 232/11 R, Rn. 16, juris; Bayerisches LSG, Urteil vom 27. Januar 2016, Az. L 12 KA 69/14, Rn. 25, juris; Pawlita in: Schlegel/Voelzke, jurisPK-SGB V, 4. Aufl., § 95 SGB V (Stand: 09.07.2021), Rn. 269; abweichend Teile der Rechtsprechung und Literatur, insb. SG Nürnberg, Urteil vom 09. April 2014, Az. S 1 KA 2/14, juris (aufgehoben), zustimmend hierzu Plagemann, Entscheidungsbesprechung zum vorgenannten Urteil, FDSozVR 2014, 358349.

7 BSG, Urteil vom 14. Dezember 2011, Az. B 6 KA 33/10 R, Rn. 19, juris; KassKomm/Rademacker, 114. EL Mai 2021, SGB V § 95 Rn. 58; Becker/Kingreen/Joussen, 7. Aufl. 2020, SGB V 99 Rn. 9. 
tätig ist, ist einerseits nicht erforderlich, andererseits auch nicht ausgeschlossen. "8

Die Stellung des ärztlichen Leiters geht zudem mit Weisungsfreiheit im Hinblick auf medizinische Fragestellungen einher, um nach der gesetzgeberischen Intention nichtmedizinische Einflussnahme durch Dritte, auch durch Träger und Geschäftsführung, auf vertragsärztliche Abläufe innerhalb des MVZ weitgehend auszuschließen. ${ }^{9}$ Dies ergibt sich ausdrücklich aus der Gesetzesbegründung zum GKV-VStG ${ }^{10}$ :

„Durch den neuen Satz 3 wird vorgegeben, dass der ärztliche Leiter in dem medizinischen Versorgungszentrum selbst als angestellter Arzt oder als Vertragsarzt tätig sein muss und in medizinischen Fragen keinen Weisungen unterliegen darf. Hierdurch wird die sich aus dem ärztlichen Berufsrecht ergebende Therapie- und Weisungsfreiheit gewährleistet, denn nur ein ärztlicher Leiter, der in die Organisations- und Versorgungsstrukturen des medizinischen Versorgungszentrums eingebunden ist, hat tatsächlich Einwirkungsmöglichkeiten auf die dortigen Abläufe und kann sicherstellen, dass ärztliche Entscheidungen unabhängig von sachfremden Erwägungen getroffen werden." ${ }^{11}$

In - früher regelhaft vorliegenden - fachgebietsübergreifenden MVZ beschränkt sich die ärztliche Leitung dabei allein auf das jeweilige Fachgebiet des ärztlichen Leiters, sodass eine Bestellung mehrerer ärztlicher Leiter bei Vorliegen mehrerer Fachgebiete in einem MVZ erforderlich sein kann. ${ }^{12}$

$8 \quad$ BSG, Urteil vom 14. Dezember 2011, Az. B 6 KA 33/10 R, Rn. 19, juris.

9 Ladurner et al., Rechtsgutachten - Stand und Weiterentwicklung der gesetzlichen Regelungen zu medizinischen Versorgungszentren, S. 98, 123, aufrufbar unter https://www.bun desgesundheitsministerium.de/fileadmin/Da teien/5_Publikationen/Ministerium/Berichte/ Stand_und_Weiterentwicklung_der_gesetzli chen_Regelungen_zu_MVZ.pdf, letzter Abruf am 01.09.2021.

10 Gesetz zur Verbesserung der Versorgungsstrukturen in der gesetzlichen Krankenversicherung vom 22.12.2011, BGBI. 2011 I, S. $2983 \mathrm{ff}$.

11 BT-Drucks. 17/6906, S. 70.

12 BT-Drucks. 17/6906, S. 117; Kyrill/Krapohl, GesR 2013, 705, 707.

\section{Aufgabenbereiche des ärztlichen Leiters}

Die Person des ärztlichen Leiters trifft nach der Rechtsprechung des BSG „zwar keine fachliche Verantwortung für jede einzelne Behandlungsmaßnahme, wohl aber die Verantwortung für die ärztliche Steuerung der Betriebsabläufe und eine Gesamtverantwortung gegenüber der KÄV." 13 Der ärztliche Leiter hat danach die insgesamt ordnungsgemäße Erfüllung der Anforderungen an die vertragsärztliche Versorgung im MVZ zu gewährleisten. ${ }^{14}$ Die genaue Ausgestaltung des Rechte- und Pflichtenspektrums des ärztlichen Leiters bestimmt sich zwar grundsätzlich anhand des konkreten Anstellungsvertrags bzw. der Zusatzvereinbarung zum Arbeitsvertrag. Im Folgenden sollen gleichwohl die allgemeinen und zentralen Verantwortungsbereiche beschrieben werden: Einerseits der medizinisch-ärztliche Aufgabenbereich, andererseits der abrechnungstechnische Verantwortungsbereich gegenüber der $\mathrm{KV}$.

\section{Allgemeiner Aufgabenbereich}

Primäre Aufgabe des ärztlichen Leiters in seiner dem Wortsinn seiner Position entsprechenden Funktion ist die steuernde Leitung der vertragsärztlichen Abläufe innerhalb des MVZ und die Sicherung des Behandlungsstandards. Um diese Aufgabe ausfüllen zu können, verfügt der ärztliche Leiter i. d. R. über ein Weisungsrecht im Rahmen seiner eigenen Fachkompetenz hinsichtlich der ihm unterworfenen angestellten Ärzte des MVZ - entsprechende Weisungsrechte gegenüber weiteren Beschäftigten im MVZ sind regelmäßig vertraglich ausgestaltet. ${ }^{15}$ In Abwesenheit eines entsprechenden Weisungsrechts trifft den ärztlichen Leiter jedenfalls regelhaft die Pflicht, den Dienstvorgesetzten - genauer den Träger über die Geschäftsführung - über die mögliche Verletzung (vertrags-)ärztlicher Pflichten durch angestellte Ärzte zu unterrichten, sodass dieser wiederum sein Direktionsrecht ausüben kann. ${ }^{16}$

13 BSG, Urteil vom 14. Dezember 2011, Az. B 6 KA 33/10 R, Rn. 18, juris.

14 Becker/Kingreen/Joussen, 7. Aufl. 2020, SGB V § 95 Rn. 9.

15 Möller in Dahm/Möller/Ratzel, Kap. XIII Rn. 54; Kyrill/Krapohl, GesR 2013, 705, $707 \mathrm{f}$.

16 Kyrill/Krapohl, GesR 2013, 705, 709
Den ärztlichen Leiter treffen in diesem Rahmen gewisse Aufsichtspflichten, da er allein oder mit kooperativ zusammenwirkenden weiteren ärztlichen Leitern des MVZ die fachliche Gesamtaufsicht führt und die Verantwortung für alle im MVZ angestellten Ärzte und Fachrichtungen und damit für die gesamte ärztliche Versorgung in dem MVZ trägt. ${ }^{17}$ Aus den Anstellungsverträgen bzw. Zusatzvereinbarungen zu den Arbeitsverträgen ergibt sich dabei in der Regel eine Verpflichtung des ärztlichen Leiters zur Gewährleistung der Einhaltung der vertragsärztlichen Vorgaben aller seiner Verantwortung unterworfenen Mitarbeiter. Aufgrund dessen trifft den ärztlichen Leiter die grundsätzliche Verpflichtung zur Überprüfung der Einhaltung der qualitativen Voraussetzungen der vertragsärztlichen Leistungserbringung im gesamten MVZ.

\section{Stellung im Rahmen der Abrechnung gegenüber der KV}

Die Honorarverteilungsmaßstäbe der Kassenärztlichen Vereinigungen (HVM) erfordern regelmäßig die Unterzeichnung der Sammelbescheinigungen im Rahmen der Quartalsabrechnung durch den ärztlichen Leiter. ${ }^{18}$ Der HVM der KV Nordrhein ${ }^{19}$ sieht hierzu in $\S 1$ Abs. 5 exemplarisch vor:

„[... In der Sammelerklärung ist durch Unterschrift zu bestätigen, dass der Unterzeichner die Verantwortung für die Erfüllung $\operatorname{der} A b$ rechnungsvoraussetzungen trägt, weil er sie selbst erfüllt oder sich von deren Erfüllung persönlich überzeugt hat. [...] Bei einem Medizinischen Versorgungszentrum (MVZ) und bei Krankenhäusern ist die Unterschrift des ärztlichen Leiters bzw. Leitenden Arztes erforderlich. [...]"

Durch seine Unterschrift bestätigt der ärztliche Leiter lediglich, dass er die Verantwortung für die Erfüllung der Abrechnungsvoraussetzungen übernimmt, da er sie selbst erfüllt oder sich von der Erfüllung persönlich überzeugt hat - dies umfasst jedoch nicht, dass er gegenüber der zuständigen KV für die Vollständiakeit und Richtiakeit der Erklä-

17 NK-MedizinR/Steinmeyer SGB V § 95 Rn. 19; Becker/Kingreen/Joussen, 7. Aufl. 2020, SGB V §95 Rn. 9.

18 Kyrill/Krapohl, GesR 2013, 705, 709.

19 HVM der KV Nordrhein, Endfassung III/2021, S. 2. 
rung verantwortlich ist und für eine fehlerhafte Abrechnung persönlich haften oder in Regress genommen werden könnte. ${ }^{20} \mathrm{Au}$ ßerdem trifft den ärztlichen Leiter nicht die Pflicht zur Sicherstellung der ordnungsgemäßen Abrechnung der in dem MVZ erbrachten vertragsärztlichen Leistungen, indem er zu überprüfen hätte, dass die Angaben auf den eingereichten Quartalsabrechnungen im Einzelfall zutreffend sind. ${ }^{21}$

Die Verantwortung für Vollständigkeit und Richtigkeit der Sammelerklärung trifft das MVZ als solches. Das BSG führte hierzu in einem richtungsweisenden Urteil aus dem Jahr 2011 aus:

„Für die organisatorischen Abläufe, insbesondere den Einsatz der Ärzte und für die Korrektheit der Abrechnung, ist das MVZ selbst verantwortlich [...]. Das MVZ ist gegenüber den Krankenkassen und der KÄV für Auswahl und Einsatz der Ärzte sowie für die Leistungsabrechnung selbst verantwortlich. Ihm obliegt die Überprüfung [... der] Korrektheit der Leistungsabrechnung und die Wirtschaftlichkeit der Behandlungen und Verordnungen sowie auch die Abgabe einer wahrheitsgemäßen Abrechnungssammelerklärung. Diese Verantwortung ist unteilbar und nicht delegierbar, sodass das MVZ gegenüber den Institutionen der vertragsärztlichen Versorgung nicht auf ein eventuelles Fehlverhalten der dort tätigen Ärzte verweisen könnte. Der Verminderung der Verantwortung des einzelnen Arztes korrespondiert die volle Verantwortung des MVZ für die korrekte Organisation der Behandlung und für die Leistungsabrechnung. Mit diesen Zuständigkeiten ist der zentrale Verantwortungsbereich des MVZ beschrieben; die Verantwortung für die organisatorischen Abläufe und für die Leistungsabrechnung kennzeichnen den Kern der Aufgaben des MVZ, sie stehen nicht wie beim Vertragsarzt neben der Aufgabe der Patientenbehandlung. "22

20 Kyrill/Krapohl, GesR 2014, 6, 9 ff.

21 Kritisch zur Reichweite der Garantiefunktion Hartmannsgruber in: Weiterdenken: Recht an der Schnittstelle zur Medizin, Festschrift für Hermann Plagemann, S. 373, 379 ff.; Möller, jurisPR-MedizinR 5/2021 Anm. 1; zurückhaltend Cansun-Labenski in: MAH Medizinrecht, Clausen/Schroeder-Printzen, 3. Auflage 2020, $\S 10$ Medizinische Versorgungszentren Rn. 79.

22 BSG, Urteil vom 21. März 2012, Az. B 6 KA 22 11 R, BSGE 110, 269-287, SozR 4-2500 § 95 $\mathrm{Nr} 24, \mathrm{Rn} .26-28$.
Aufgrund des Vorgenannten wird das MVZ nicht durch Bestellung eines ärztlichen Leiters von seiner Verantwortung für die ordnungsgemäße Erfüllung der vertragsärztlichen Vorschriften befreit - die Verantwortung des MVZ als vertragsarztrechtliche Entität in einer wahrheitsgemäßen Abrechnungssammelerklärung mündenden Abrechnung gegenüber der KV ist unteilbar und nicht auf einen ärztlichen Leiter delegierbar. ${ }^{23}$

Das MVZ, das im Falle etwaiger Regressforderungen der zuständigen KV zur Rückzahlung verpflichtet ist, ist für ein Fehlverhalten der angestellten Ärzte im Rahmen der Abrechnung allein verantwortlich und muss sich dieses zurechnen lassen. Honorarrückforderungsansprüche wegen fehlerhafter Abrechnung richten sich nicht gegen den ärztlichen Leiter, da auch die Honoraransprüche für die erbrachten ärztlichen Leistungen dem MVZ als Zulassungsinhaber und nicht dem ärztlichen Leiter zustehen. Das Betriebsrisiko und die Organisationshoheit verbleiben bei der MVZ-Trägergesellschaft. Mit vorstehendem Urteil hat das BSG etwaige Versuche einer MVZ-Trägergesellschaft, die Verantwortung auf den ärztlichen Leiter zu verlagern, für unzulässig erklärt. ${ }^{24}$

\section{Haftung des ärztlichen Leiters im Innenverhältnis}

Eine Haftung des ärztlichen Leiters kann allenfalls im Innenverhältnis gegenüber dem Arbeitgeber bestehen, wenn ihm schuldhafte Sorgfaltspflichtverletzungen in seiner Funktion als ärztlicher Leiter nachgewiesen werden können, die auf der Verletzung der arbeitsvertraglich vereinbarten Aufgaben beruhen. ${ }^{25}$ Diese Haftung ist dabei regelmäßig aufgrund der Grundsätze der privilegierten Arbeitnehmerhaftung sowie arbeitsvertragliche Haftungsbeschränkungen begrenzt.

Etwaige Fehler in der Quartalsabrechnung führen nicht zu einer Haftung des ärztlichen Leiters im Innenverhältnis gegenüber dem MVZ, wenn der ärztliche Leiter die

23 Kyrill/Krapohl, GesR 2013, 705, 710.

24 BSG, Urteil vom 21. März 2012, Az. B 6 KA 22/ 11 R, BSGE 110, 269-287, SozR 4-2500 §95 Nr 24, Rn. 26-28.

25 Kyrill/Krapohl, GesR 2014, 6, 9.
Sammelbescheinigung ohne Kenntnis der Mängel unterschreibt. ${ }^{26}$ Eine detaillierte Überprüfung der Quartalsabrechnung vor Abgabe ist daher wohl nicht zu verlangen und wäre auch aufgrund des Umfanges der in mittleren und großen MVZ abgerechneten Positionen unzumutbar - insbesondere, da ärztliche Leiter neben Ihrer Leitungsstellung selbst ärztlich tätig werden. Das MVZ selbst hat über die Geschäftsführung insoweit für die ordnungsgemäße Abrechnung zu sorgen, die durch den ärztlichen Leiter lediglich zu unterzeichnen, jedoch inhaltlich nicht zu überprüfen ist.

\section{Disziplinargewalt der zuständigen Kassenärztlichen Vereinigung gegenüber dem ärztlichen Leiter}

Grundsätzlich unterliegt der ärztliche Leiter eines MVZ der Disziplinargewalt der KV, da er als Angestellter oder Vertragsarzt an der vertragsärztlichen Versorgung teilnimmt. Trifft dies zu, kann der Arzt im Rahmen der Disziplinargewalt der KV durch entsprechende Disziplinarmaßnahmen für Fehlverhalten sanktioniert werden. ${ }^{27}$ Das BSG stellte hierzu instruktiv fest:

„Ein Fehlverhalten im vertragsärztlich relevanten Bereich könnte disziplinarisch zwar gegenüber den im MVZ tätigen Vertragsärzten oder angestellten Ärzten, die, soweit sie einen vollen oder hälftigen Versorgungsauftrag wahrnehmen, nach § 77 Abs 3, § 95 Abs 3 Satz 2 $S G B V$ Mitglied der für den Vertragsarztsitz des MVZ zuständigen KÄV werden, geahndet werden. Sofern ein solcher personenbezogener Durchgriff nicht möglich ist, kann ein Fehlverhalten auch dem Versorgungszentrum als solchem zugerechnet werden. Das MVZ als zugelassener Leistungserbringer muss sich die Pflichtverletzungen der bei ihm in die vertragsärztliche Versorgung eingebundenen Ärzte zurechnen lassen [...]."28

26 Kyrill/Krapohl, GesR 2014, 6, 11.

27 Krauskopf/Gerlach, 111. EL Mai 2021 Rn. 23, SGB V §95 Rn. 23

28 BSG, Urteil vom 14. Dezember 2011, Az. B 6 KA 33/10 R, Rn. 20, juris. 
Die Disziplinarmaßnahmen können sich dabei - tat- und schuldangemessen - auf eine Verwarnung oder ein Ordnungsgeld beschränken, bei schwerwiegenden persönlichen Fehlleistungen kann auch die Bestellung des betreffenden Arztes zum ärztlichen Leiter oder dessen Anstellungsgenehmigung im MVZ widerrufen werden. ${ }^{29}$

Die Reichweite der disziplinarischen Gewalt der KV über die ärztlichen Leiter verdeutlicht anschaulich eine aktuelle Entscheidung des SG München. ${ }^{30}$ Der Kläger ist ärztlicher Leiter eines MVZ, dass mit einem fachgleichen nahegelegenen MVZ zusammenarbeitete. Im Rahmen einer Plausibilitätsprüfung stellte die KV die Implausibilität der Abrechnung fest und erließ einen Rückforderungsbescheid. Parallel ging sie disziplinarisch gegen den Kläger vor, dieser habe die vertragsärztliche Pflicht zur peinlich genauen Abrechnung verletzt. Unter seiner ärztlichen Aufsicht und Verantwortung seien erbrachte Leistungen nicht ordnungsgemäß dokumentiert und die Dokumentation der KV auf Anforderung nicht zur Prüfung vorgelegt worden, die MVZ hätten als Praxisgemeinschaft rechtsmissbräuchlich ohne Genehmigung wie eine überörtliche BAG gem. §33 Abs. 3 ÄrzteZV zusammengearbeitet, interne Überweisungen seien vielfach medizinisch nicht nachvollziehbar gewesen und es sei zu rechtsmissbräuchlichen Doppelbehandlungen gekommen. Der Kläger habe seine Sorgfaltspflicht als ärztlicher Leiter schuldhaft verletzt, dieses Fehlverhalten sei mit einer Geldbuße von $8000 €$ zu sanktionieren. Er könne sich insbesondere nicht darauf berufen, die Leistungen nicht selbst erbracht zu haben, da ihn als ärztlichen Leiter die Gesamtverantwortung hinsichtlich der Abrechnung treffe und er die Abrechnung

29 Pawlita in: Schlegel/Voelzke, jurisPK-SGB V, 4. Aufl., §95 SGB V (Stand: 09.07.2021), Rn. 273.1; Kyrill/Krapohl, GesR 2013, 705, 710 f.; Ladurner et al., Rechtsgutachten Stand und Weiterentwicklung der gesetzlichen Regelungen zu medizinischen Versorgungszentren (MVZ), S. 112, aufrufbar unter https://www.bundesgesundheitsministerium. de/fileadmin/Dateien/5_Publikationen/Minis terium/Berichte/Stand_und_Weiterentwick lung_der_gesetzlichen_Regelun gen_zu_MVZ.pdf, letzter Abruf am 01.09.2021.

30 SG München, Gerichtsbescheid vom 22. Januar 2021, Az. S 38 KA 165/19. in Hinblick auf Korrektheit und Vollständigkeit der Dokumentation hätte überprüfen müssen. Der Kläger war der Auffassung, ihm könne kein Vorwurf gemacht werden, insbesondere könne er nicht jeden Abrechnungsvorgang prüfen. Die Pflichtverletzungen der ihm nachgeordneten angestellten und Vertragsärzte träfen diese selbst und seien ihm jedenfalls nicht aufgrund seiner Funktion als ärztlicher Leiter zurechenbar.

Nach Auffassung des SG München war das Vorgehen der KV nicht zu beanstanden, es haben sanktionsbedürftige Pflichtverletzungen des ärztlichen Leiters gelegen. ${ }^{31}$ In den durch die KV erhobenen Vorwürfen liege eine Verletzung der Pflicht zur peinlich genauen Abrechnung als Grundpflicht des Vertragsarztsystems. Die Pflichtverletzungen auch wenn nicht in personam des ärztlichen Leiters begangen - seien dem Kläger auch zuzurechnen, da dieser als ärztlicher Leiter des MVZ eine besondere Pflichtenstellung im Sinne einer Gesamtverantwortung gegenüber der KV einnehme. Die Kernaufgaben des MVZ stellen nach Auffassung des Gerichts die korrekte Organisation der Behandlung und der Leistungsabrechnung dar; diese Aufgaben würden durch die Person des ärztlichen Leiters wahrgenommen. Der ärztliche Leiter garantiere mit seiner Unterschrift unter der Sammelerklärung,

„dass die Abrechnung ordnungsgemäß, d. h. auch vollständig entsprechend der Leistungslegende erbracht wurde. Daraus folgt, dass der ärztliche Leiter letztendlich die Gesamtverantwortlichkeit gegenüber der [KV] für die von den angestellten Ärzten erbrachten Leistungen trägt. “32

Ein Durchgriff auf die Person des ärztlichen Leiters im Rahmen von Disziplinarmaßnahmen sei - auch unter Rückgriff auf die ständige Rechtsprechung des BSG - zulässig und notwendig, aufgrund der Gesamtverordnung des ärztlichen Leiters bestehe auch keine Notwendigkeit, vorrangig gegen angestellte Ärzte - die Pflichtverletzungen begangen haben - im MVZ und

31 SG München, Gerichtsbescheid vom

22. Januar 2021, Az. S 38 KA 165/19, Rn. 27, juris.

32 SG München, Gerichtsbescheid vom 22. Januar 2021, Az. S 38 KA 165/19, Rn. 37, juris. nur subsidiär gegen den ärztlichen Leiter vorzugehen. ${ }^{33}$

Die Entscheidung des SG ist kritisch zu hinterfragen; sie weitet das persönliche Haftungsrisiko des ärztlichen Leiters in erheblichem Maße aus. Fraglich ist insbesondere der durch das SG angenommene Erklärungsgehalt der Unterschrift des ärztlichen Leiters unter der Sammelbescheinigung im Rahmen der Abrechnung. Gegen die Auffassung des SG, der ärztliche Leiter bestätige damit Richtigkeit und Vollständigkeit jedes einzelnen Abrechnungspunktes, spricht die tägliche Praxis des durchschnittlichen MVZ. Dem ärztlichen Leiter ist es i. d. R. weder möglich noch zuzumuten, die Einhaltung jeder einzelnen Abrechnungsvoraussetzung hinsichtlich jeder einzelnen abzurechnenden Position durch jeden einzelnen in dem MVZ tätigen Arzt zu überwachen und hierfür zu garantieren. Zudem wird diese Ausweitung des persönlichen Haftungsrisikos nach der vertragsgestalterischen Erfahrung in der Regel nicht ausreichend monetär kompensiert.

\section{Zusammenfassung}

Der ärztliche Leiter nimmt im MVZ eine herausgehobene Schlüsselstellung ein - er verantwortet die medizinisch-ärztliche Organisation und Operation des MVZ und stellt im Rahmen der Leistungserbringung die Einhaltung der vertragsärztlichen Vorschriften gegenüber der KV im Sinne einer eher symbolischen Gesamtverantwortung sicher. ${ }^{34}$ Im Rahmen der Abrechnung tritt er gegenüber der zuständigen KV für das MVZ in Erscheinung.

Das persönliche Haftungsrisiko eines ärztlichen Leiters war und ist grundsätzlich als gering und gut kalkulierbar einzuschätzen. Da zwischen ärztlichem Leiter und KV kein unmittelbares Rechtsverhältnis besteht, kann der ärztliche Leiter für etwaige Fehler in der Abrechnung oder vertragsärztliche Pflichtverletzungen im Wege eines Regresses nicht persönlich in Anspruch genom-

33 SG München, Gerichtsbescheid vom 22. Januar 2021, Az. S 38 KA 165/19, Rn. 37, juris unter Bezugnahme auf BayLSG, Urteil vom 27. Januar 2016, Az. L 12 KA 69/14.

34 Kyrill/Krapohl, GesR 2014, 6, 11. 
men werden. ${ }^{35}$ Das BSG lehnt darüber hinaus jede Verlagerung dahingehender haftungsrelevanter Pflichten auf den ärztlichen Leiter als nicht teilbar und nicht delegierbar ab. Das Haftungsrisiko gegenüber dem Arbeitgeber aufgrund der Verletzung arbeitsvertraglich vereinbarter Pflichten des ärztlichen Leiters ist - auch vor dem Hintergrund arbeitsrechtlicher und -vertraglicher Haftungsprivilegierungen - als gering zu bewerten, sofern dieser die ihm vertraglich auferlegten Pflichten ordnungsgemäß erfüllt.

Die Entscheidung des SG München verdeutlicht jedoch die latente Gefahr der disziplinarischen Sanktionierung durch die KVen, auch bei nicht durch den ärztlichen Leiter begangenen, sondern ihm zugerechneten Pflichtverletzungen angestellter Ärzte. Die Entscheidung ist insofern geeignet, ärztliche Leiter zu bewegen, die Einhaltung der vertragsarztrechtlichen Vorschriften durch angestellte Ärzte im MVZ genauer zu prüfen. Vor diesem Hintergrund erscheint die Übernahme der mit der Position als ärztlicher Leiter einhergehenden Verantwortung zunehmend unattraktiver. Da es sich jedoch um eine erstinstanzliche Entscheidung handelt bleibt abzuwarten, wie insbesondere die Landessozialgerichte und das BSG vergleichbare Fälle bewerten werden. Vorliegend ist ergänzend darauf hinzuweisen, dass das Verfahren im Wege eines Vergleichs zwischen Kläger und beklagter KV durch Zahlung eines deutlich herabgesetzten Ordnungsgeldes hätte vermieden werden können, hätte der Kläger den bereits geschlossenen Vergleich nicht widerrufen. ${ }^{36}$

Abschließend bleibt anzumerken, dass Vertragsärzte in Einzelpraxis und Berufsausübungsgemeinschaften in Bezug auf die Anforderungen an die ärztliche Leitung gegenüber MVZ weiterhin strukturell benachteiligt werden, sodass eine Haftungsverschärfung gegenüber dem ärztlichen Leiter eines MVZ angezeigt sein könnte. Ein Vertragsarzt, der nicht in einem MVZ tätig ist, hat nach §14a Abs. 1 S. 1 BMV-Ä sicherzustellen, dass er „die Arztpraxis persönlich leitet". Aufgrund dessen ist seine Möglichkeit zur Anstellung weiterer Ärzte gem. §14a Abs. 1 S. 2 BMV-Ä auf maximal drei weitere vollzeitbeschäftigte Ärzte beschränkt. Bei Vertragsärzten, die überwiegend medizinisch-technische Leistungen erbringen, darunter Fachärzte für Radiologie, wird die persönliche Leitung nach §14a Abs. 1 S. 3 BMVÄ auch bei der Beschäftigung von bis zu vier vollzeitbeschäftigten Ärzten vermutet. Der Grund für diese Beschränkung in der Beschäftigung angestellter Ärzte liegt darin, dass eine persönliche Aufsichts- und Wei- sungsmöglichkeit durch den anstellenden Vertragsarzt gegeben sein muss. Eine entsprechende Begrenzung besteht für MVZ nicht, so dass insbesondere bei der Beschäftigung einer größeren Anzahl von Ärzten die Aufsichtsmöglichkeiten durch den ärztlichen Leiter beschränkt sein dürften. Aufgrund der unbegrenzten Skalierbarkeit der Anstellungen im MVZ könnte daher - zur Sicherstellung der tatsächlichen und ordnungsgemäßen Erfüllung der Versorgungsaufträge des MVZ - eine Haftungsausweitung des ärztlichen Leiters als verantwortlicher Schlüsselfigur angezeigt sein.

Prof. Dr. Peter Wigge

Rechtsanwalt

Fachanwalt für Medizinrecht

Florian Dawe

Dipl.-Jur. (WWU Münster) B. A. General

Management

Wissenschaftlicher Mitarbeiter

Rechtsanwälte Wigge

Scharnhorststraße 40

48151 Münster

Telefon: (0251) 53 595-0

Telefax: (0251) 53 595-99

E-Mail: kanzlei@ra-wigge.de www.ra-wigge.de
36 SG München, Gerichtsbescheid vom 22. Januar 2021, Az. S 38 KA 165/19, Rn. 15, juris. 